\title{
Necessidades de ações educativas-terapêuticas em um serviço de diálise renal no Brasil
}

\author{
Larissa Padilha Fernandes ${ }^{1}$, Karine Yara Mota Marins ${ }^{1}$, Hercules de Oliveira Carmo ${ }^{2}$, Samira Rodrigo dos \\ Santos Silva ${ }^{3}$, Silvia Maria de Carvalho Farias ${ }^{4}$, Clinton Fábio Gomes da Silva ${ }^{5}$
}

${ }^{1}$ Enfermeira. Escola Superior de Cruzeiro. São Paulo - Brasil.

${ }^{2}$ Enfermeiro. Doutorando em Gerenciamento em Enfermagem e serviços de saúde. Universidade de São Paulo USP-e Docente em Enfermagem na Escola Superior de Cruzeiro. São Paulo - Brasil.

${ }^{3}$ Enfermeira e Pedagoga. Especialista em Enfermagem em Centro Cirúrgico pela Universidade de São Paulo USP-e Docente em Enfermagem na Escola Superior de Cruzeiro. São Paulo - Brasil.

${ }^{4}$ Enfermeira. Mestra em Unidade de Terapia Intensiva pela Sociedade Brasileira de Terapia Intensiva (SOBRATI/ IBRATI). Coordenadora e Docente em Enfermagem na Escola Superior de Cruzeiro. São Paulo. Brasil.

${ }^{5}$ Enfermeiro. Especialista em Vigilância em Saúde. Faculdade de Medicina de São José do Rio Preto - FAMERP. São Paulo. Brasil.

\section{Resumo}

Introdução: As ações educativas frequentemente são subestimadas pelos profissionais atuantes em serviços de hemodiálise (HD), mesmo sabendo que elas são imprescindíveis no processo terapêutico. Objetivo: investigar as dúvidas e/ou necessidades das pessoas com DRC e a existência de um programa educacional em um serviço de HD.

Método: estudo descritivo e exploratório, com abordagem qualitativa desenvolvido em uma Unidade de Terapia Dialítica, no Brasil. Participaram 30 pessoas com DRC em tratamento HD. Os dados e as elocuções foram coletados por meio de entrevistas individuais, com roteiro semiestruturado e analisado, através de categorias temáticas.

Resultado: não se evidenciou predominância para categoria sexo, sendo $15(50 \%)$ para ambos, a faixa etária variou entre 40 a 93 anos. Em relação ao tempo que realiza a HD predomínio foi entre 1 e 5 anos (53\%). Os participantes revelaram a ausência (20\%) e/ou conhecimento

Correspondencia:

Hercules de Oliveira Carmo

Escola Superior de Cruzeiro "Prefeito Amilton Vieira Mendes" Rua Doutor José Rodrigues Alves Sobrinho

$n^{\circ} 499 / 500$ Vila Suely

12711-690 - Cruzeiro, SP - Brasil

E-mail: hercules.enf@usp.br limitado (80\%) sobre as questões que envolvem a DRC, identificaram-se através dos discursos, nove necessidades de esclarecimentos, principalmente sobre como se desenvolve $(23 \%)$, diagnóstico precoce $(8 \%)$, prevenção $(8 \%)$ e tratamentos (33\%). Apontaram (40\%) que, o profissional de enfermagem foi o precursor na prestação de informação relativo à doença e autocuidado.

Conclusão: A função de educador é um dos domínios fundamentais dos cuidados de enfermagem, porém, é sabido das dificuldades que tais profissionais enfrentam para viabilizar tal competência. Faz se necessária uma reorientação da prática educacional neste serviço, com 0 objetivo de ofertar melhor esclarecimentos sobre a DRC, e consequentemente, uma melhor qualidade de vida a estas pessoas.

PALAVRAS-CHAVE: insuficiência renal; diálise renal; educação em saúde; unidades hospitalares de hemodiálise.

\section{Needs of Educational-Therapeutic Actions in a Renal Dialysis Service in Brazil}

\section{Abstract}

Introduction: Educational actions are often underestimated by professionals working in hemodialysis (HD) 
units, even though they are essential in the therapeutic process.

Objective: To determine the doubts and/or needs of people with chronic kidney disease (CKD) and the implementation of an educational program in a HD unit.

Method: A descriptive and exploratory study with a qualitative approach developed in a Dialysis Therapy Unit in Brazil. Thirty people with CKD on HD treatment were enrolled. Data and elocutions were collected through individual interviews, with a semi-structured questionnaire and analyzed through thematic categories.

Results: No predominance was found for the sex category, $15(50 \%)$ for both. The age range ranged from 40 to 93 years. Regarding HD time, the prevalence was between 1 and 5 years (53\%). Participants revealed the absence (20\%) and / or limited knowledge (80\%) on the issues involving CKD. Nine needs for clarification were identified through the speeches: mainly about how it develops $(23 \%)$, early diagnosis $(8 \%)$, prevention $(8 \%)$ and treatments (33\%). They pointed out (40\%) that the nursing professional was the forerunner in providing information regarding the disease and self-care. Conclusion: One of the fundamental domains of nursing care is the role of educator. However, these professionals face many difficulties to enable such competence. A reorientation of the educational practice in this service is required, with the purpose of offering better clarifications on the CKD, and consequently, a better quality of life to these people.

KEYWORDS: renal insufficiency; renal dialysis; health education; hemodialysis hospital units.

\section{Introdução}

A Doença Renal Crônica (DRC) é uma lesão renal, caracterizada por anormalidades estruturais ou funcionais, com ou sem diminuição da taxa de filtração glomerular (TFG), caracterizada como grave e grande problema de saúde pública, devida a alta morbidade e mortalidade. Além disso, repercute negativamente para a economia no sistema de saúde, causando ainda mais impacto social e pessoal. 0 aumento progressivo de sua incidência e prevalência, associado a prognósticos não favoráveis, faz com que a DRC, seja apontada dentre outras a "epidemia deste milênio" 1 .
Estima-se que há cerca de aproximadamente 1,5 milhão de brasileiros com DRC ${ }^{2}$, e de acordo com o Sistema de Informação Hospitalar (SIH) do Ministério da Saúde (MS) do Brasil, a taxa de mortalidade por insuficiência renal, com base na Classificação Internacional de Doenças (CID-10), de 2010 a 2015 é alarmante e crescente os dados, sendo: $11,67 \%$ (2010); 12,03\% (2011); $12,14 \%$ (2012); 12,27\% (2013); $12,62 \%$ (2014); e 13,13\% (2015) 3 .

Infelizmente a DRC é silenciosa, a pessoa só começa a perceber, quando apresenta alguma alteração renal, e inicia o aparecimento dos sintomas urêmicos, ou seja, quando os rins perderam aproximadamente $50 \%$ de sua função $0^{4}$. No estágio final da DRC, faz se necessária uma intervenção terapêutica, denominada diálise renal, para o resto da vida ou até o transplante renal ${ }^{5}$. Dados do MS, em um censo realizado no ano de 2015, revela que mais de 100 mil pessoas fazem diálise, existem 750 unidades de diálise cadastradas ${ }^{6}$ e os gastos com a terapia renal de substitutiva chegam a mais dois bilhões anualmente no Brasil?.

0 método mais empregado em território brasileiro para o tratamento da DRC é a hemodiálise (HD), que remove substâncias nitrogenadas tóxicas e o excesso de água através de um circuito extracorpóreo formado por linha arterial e venosa de material sintético, e um hemodialisador $^{8}$. Nesse tratamento, a pessoa com DRC se torna obrigado a se deslocar até o serviço de diálise, em média de três vezes por semana, permanecendo aproximadamente quatro horas ligado a uma máquina9, susceptível as complicações da HD, além de ter que enfrentar as repercussões psicológicas e sociais da doença ${ }^{10}$. É frequente que as pessoas com DRC apresentarem desânimo frente à doença e ao tratamento dialítico, além de motivação escassa ou ausente para o autocuidado, principalmente pela falta ou perda da informação ${ }^{4}$.

Existem diferentes fatores que afetam a perda de informação, tais como a própria doença crônica, que com o tempo gradualmente os faz esquecer; a idade, cada vez mais avançada também desempenha um papel determinante no momento dessa perda; e a emergência, há casos que começam HD de forma urgente e não recebem as devidas orientações ${ }^{11}$. 0 enfermeiro é o profissional mais constante e próximo desta pessoa com DRC, assim deve sempre estar fornecendo cuidados e intervenções educativas, visando ajudá-lo a conviver com as novas mudanças impostas pela doença ${ }^{12}$. Uma vez que as competências do enfermeiro são agrupadas em: administrativa, assistencial, educativas e pesquisa ${ }^{8}$. 
A intervenção educacional de enfermagem propicia uma abordagem com a pessoa doente, de modo que estabelecem uma comunicação e um vínculo, facilitando a compreensão do agravo e possibilitando maior adesão ao tratamento ${ }^{13-14}$. A intervenção educacional, pode se constituir em: informações impressas com material educativo ou orientações personalizadas, individuais ou em grupos, com vistas a instrumentalizar o indivíduo para o autocuidado, como sujeito de sua terapêuti$\mathrm{ca}^{14}$. Entretanto na prática, sobra pouco tempo para as ações educativas, a prioridade é dada às ações assistências e administrativas ${ }^{15}$. As mudanças de paradigmas direcionadas as práticas educativas em saúde, ainda são incipientes e possui pequena visibilidade no cenário nacional, justificando assim, a não percepção como estratégia auxiliar e elementar no processo de cuidar ${ }^{16}$.

Neste contexto, pesquisas sobre dados epidemiológicos e com opiniões das pessoas com DRC, representam uma contribuição valiosa para a assistência de enfermagem, pois os resultados obtidos contribuem para a adoção de estratégias, que priorizam a sua adesão, tratamento e autocuidado. Visto a tamanha responsabilidade do enfermeiro frente à pessoa com DRC, este estudo objetivou em investigar junto às pessoas com Doença Renal Crônica suas dúvidas e/ou necessidades relacionados à doença, o tratamento e autocuidado e a existência de um programa educacional em um serviço de HD.
Os dados e as elocuções foram coletados por meio de entrevistas individuais, com roteiro semiestruturado, gravadas na integra, por equipamento de áudio formato de MP4, na própria unidade de Terapia Dialítica, em períodos matutinos e vespertinos e agendamento prévio com a instituição. Os participantes receberam informações sobre o estudo e objetivo, assegurado que o conteúdo coletado somente seria utilizado mediante autorização e assinatura do Termo de Consentimento Livre e Esclarecido.

Foram elaborados dois instrumentos pelo grupo de pesquisa, sendo um primeiro para traçar o perfil (sexo, idade, estado civil, escolaridade, tempo de diagnóstico da doença, tempo de tratamento e recebimento de informação no serviço) e o segundo, um roteiro de entrevista semiestruturada, consistindo das seguintes perguntas: Fale sobre o que sabe a respeito da doença renal crônica; conte-me suas dúvidas e/ou necessidades de informações em relação a doença e/ou tratamento para doença renal crônica.

A análise do perfil foi realizada de forma descritiva, por meio de números absolutos e percentuais, apresentado em forma de tabelas, quadros e gráficos, utilizando-se, para isso, o Programa Office Excel 2010. Para análise das entrevistas, utilizou-se a análise temática ${ }^{17}$ (Figura 1), a partir do objetivo proposto.

\section{Método}

Trata-se de estudo descritivo e exploratório, com abordagem qualitativa, desenvolvido em uma Unidade de Terapia Dialítica, no interior do Estado de São Paulo, no Brasil.

A seleção do estabelecimento de saúde, foi por conveniência, sendo avaliado: a localidade da unidade, os custos da pesquisa e tempo de atuação na área. A população é

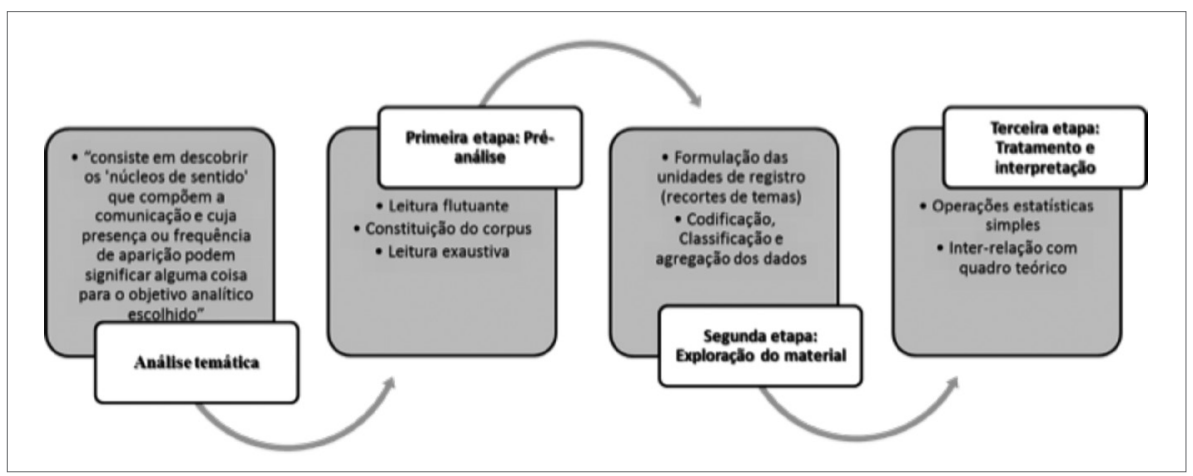

Figura 1. Percurso metodológico para análise da temática ${ }^{17}$. composta por pessoas diagnosticadas com Doença Renal Crônica, que estavam em tratamento no período de janeiro e fevereiro de 2016, tendo como critério de inclusão: possuir idade igual ou superior a 18 anos e estar em tratamento dialítico (HD) no período da coleta de dados. Os participantes ausentes na unidade, os que apresentassem agravamento do seu estado de saúde e sem condições para responder verbalmente a entrevista, não participaram do estudo.
0 projeto de pesquisa foi aprovado pelo Comitê de Ética e Pesquisa do Centro Universitário Teresa D'Ávila sob o parecer $n^{\circ} 1.181 .880$ juntamente com a instituição estudada, conforme as normas da legislação $466 / 2012^{18}$. 


\section{Resultados}

Aceitaram participar do estudo 30 pessoas com DRC. Dentro das características do perfil destes entrevistados, não se evidenciou predominância para categoria sexo, sendo $15(50 \%)$ para homens e mulheres, a faixa etária variou entre 40 a 93 anos (média de 61,46 anos e mediana de 59 anos), sendo: 06 (20\%) entre 21 a 50 anos, $12(40 \%)$ entre 51 e 60 anos, 05 (17\%) entre 61 e 70 anos, $04(13 \%)$ entre 71 e 80 anos e $03(10 \%)$ com mais de 81 anos. No estado civil, houve predomínio de 21 casados $(70 \%)$, viúvos, solteiros e divorciados apareceram mesma quantidade para cada $3(10 \%)$. Já quanto à escolaridade, 05 revelaram não ter escolaridade nenhuma ( $17 \%), 16$ possuíam ensino fundamental completo (53\%), 08 indivíduos verbalizaram ensino médio completo $(27 \%)$ e apenas 01 com ensino superior completo (3\%). Em relação ao tempo que realiza a $\mathrm{HD}$, os resultados variaram entre um mês e 13 anos, com predomínio para os que realizam entre 1 e 5 anos (53\%), seguidos dos que realizam a menos de um ano (20\%), e entre $6 \mathrm{e}$ 10 anos (17\%) (Tabela 1).

Tabela 1. Distribuição dos participantes segundo variáveis: sexo, idade, estado civil, escolaridade e tempo que realiza HD em uma Unidade de Terapia Dialítica, interior do estado de São Paulo, Brasil, 2016.

\begin{tabular}{|c|c|c|}
\hline & VARIÁVEIS & PARTICIPANTES \\
\hline Sexo & $\mathrm{N}=30$ & Percentual (\%) \\
\hline Feminino & 15 & $50 \%$ \\
\hline Masculino & 15 & $50 \%$ \\
\hline \multicolumn{3}{|l|}{ Idade } \\
\hline $20-50$ & 06 & $20 \%$ \\
\hline $51-60$ & 12 & $40 \%$ \\
\hline $61-70$ & 05 & $17 \%$ \\
\hline $71-80$ & 04 & $13 \%$ \\
\hline$>81$ & 03 & $10 \%$ \\
\hline \multicolumn{3}{|l|}{ Estado Civil } \\
\hline Solteiro/a & 03 & $10 \%$ \\
\hline Casado/a & 21 & $70 \%$ \\
\hline Separado/a & 03 & $10 \%$ \\
\hline Viúvo/a & 03 & $10 \%$ \\
\hline \multicolumn{3}{|l|}{ Escolaridade } \\
\hline Analfabetos & 05 & $17 \%$ \\
\hline Ensino Fundamental & 16 & $53 \%$ \\
\hline Ensino Médio & 08 & $27 \%$ \\
\hline Ensino Superior & 01 & $3 \%$ \\
\hline \multicolumn{3}{|l|}{ Tempo que realiza HD } \\
\hline$\geq 11$ meses & 06 & $20 \%$ \\
\hline $1-5$ anos & 16 & $53 \%$ \\
\hline $6-10$ anos & 05 & $17 \%$ \\
\hline$\leq 11$ anos & 03 & $10 \%$ \\
\hline
\end{tabular}

Fonte: elaboração própria.
Os achados a seguir (Tabela 2) referem-se à percepção da pessoa com DRC quanto ao recebimento de informações relativas à doença e tratamento. Sabe-se que a informação ou conhecimento sobre a doença e o tratamento são uns dos pressupostos da adesão, uma vez que ninguém pode aderir a determinadas recomendações se não as conhecer. Neste contexto, procuramos saber se estas pessoas com DRC se consideram informadas, quem as forneceram, em quais momentos, de que modo e de que tipo e se algumas destas informações recebidas influenciaram na adesão.

Tabela 2. Distribuição da relação entre a informação recebida e percepção dos participantes que realiza HD em uma Unidade de Terapia Dialítica, interior do estado de São Paulo, Brasil, 2016.

\begin{tabular}{lll}
\hline Domínio & Categoria & Subcategoria \\
Informação & Autopercepção $(n=30)$ & $\begin{array}{l}\text { Bem informado (9) } \\
\text { Informado (15) } \\
\text { Pouco informado (06) }\end{array}$ \\
\cline { 2 - 3 } & Utilidade ( $n=30)$ & $\begin{array}{l}\text { Utiliza da informação } \\
\text { recebida (19) } \\
\text { Utiliza parcialmente } \\
\text { a informação recebida (08) } \\
\text { Não utiliza a informação } \\
\text { recebida (03) }\end{array}$ \\
& & Pré-diálise (11) \\
Inicial (14) \\
$\begin{array}{l}\text { Periodicidade que mais } \\
\text { recebeu informação } \\
(n=30)\end{array}$ & Época natalícia e festas (05) \\
& Tipo ( $n=30)$ & Escrita (08) \\
& Oral (22) \\
\hline Membros da equipe & Médico (05) \\
$(n=30)$ & Enfermeiro (12) \\
& Nutricionista (08) \\
& Outros (05) \\
\hline
\end{tabular}

Fonte: elaboração própria.

As pessoas com DRC demonstraram interesse em compartilhar suas necessidades em esclarecimentos e dúvidas em diversas temáticas, e que certamente, diante destes discursos retratam as dificuldades vivenciadas no cotidiano entre 'serviço de saúde, doente e profissional' (Figura 1). A partir destas necessidades, foi possível categorizar os discursos em três áreas temáticas. 


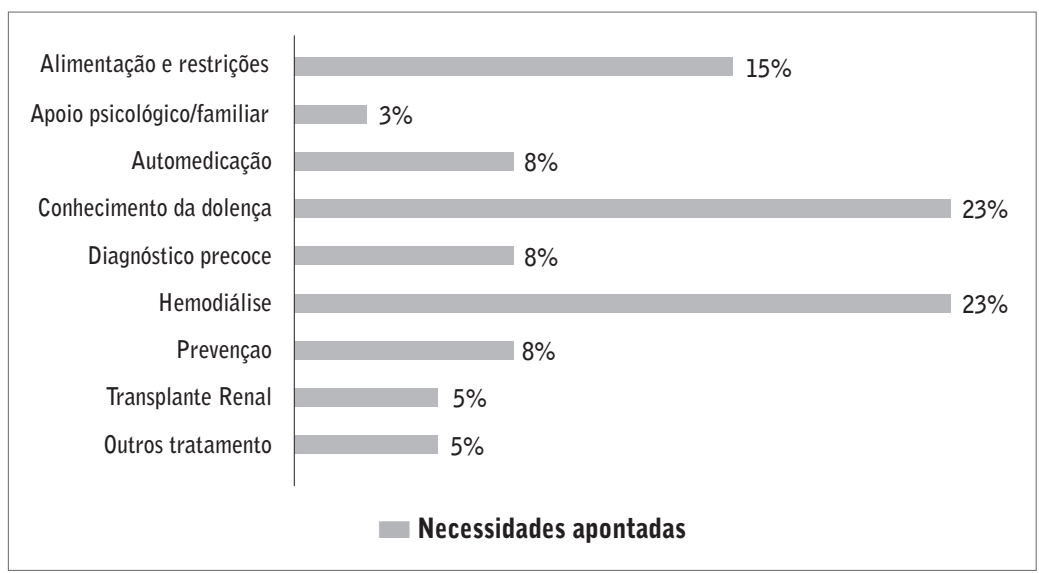

Figura 2. Distribuição das necessidades de esclarecimentos apontadas pelos participantes que realiza HD em uma Unidade de Terapia Dialítica, interior do estado de São Paulo, Brasil, 2016. $(n=30)$.

Fonte: elaboração própria.

Categoria 1 - Descobrindo a doença (como se desenvolve, diagnóstico precoce e prevenção para doença renal): Nos relatos dos participantes, identificou-se a ausência e/ou conhecimento limitado sobre as questões que envolvem a patologia, ressaltando a necessidade de identificação da anormalidade o mais cedo possível.

"É bom cuidar da saúde desde cedo (P29); 0 que é a doença e como se faz o tratamento? (P22); É importante conhecer o que é a doença e ter diagnóstico precoce (P23); É importante que as pessoas procurem o médico regularmente, para descobrir a doença no começo e tratar, e não aconteça como foi comigo, quando descobri já não tinha mais jeito (P19); As pessoas deveriam conhecer melhor a doença e saber como se previne e descobrir cedo o problema (P21)."

\section{Categoria 2 - Dia a dia da pessoa com Doença Renal}

Crônica (alimentação para portadores de insuficiência renal, apoio psicológico e familiar e automedicação): A necessidade de mudar os hábitos na alimentação, a convivência com as dores e outras rotinas, parecem serem os maiores problemas no cotidiano dos indivíduos com DRC, conforme evidenciado abaixo:

"Tem toda uma dieta regrada, e é bom segui-la para não piorar (P01); Não posso comer tudo, sigo sempre as orientações dadas pelo médico na clínica (P04); Fico inconformado por só poder comer uma concha de feijão (P11); Por que tenho que evitar comer alguns alimentos? ( $P 12) ;$ Por que a alimentação tem que ser restrita? (P26); É sempre bom ir no médico, ter uma boa alimentação, pra todos hipertensos antes que tenha que fazer HD (P27)".
"Sinto dores às vezes, mas não é qualquer remédio que posso ir tomando (P05); Depois que tive a doença só tomo remédio que o médico me recomenda (P13); Automedicação pode causar a insuficiência renal? (P24)". "É importante às pessoas saberem que, quando o paciente descobre a doença, necessita de apoio psicológico e dos familiares, porque não é fácil aceitar o diagnóstico (P19)".

\section{Categoria 3 - Conformidade e esperança} (HD, transplante renal e outros tipos de tratamentos possíveis): Na avaliação do conhecimento e necessidade de esclarecimento, os resultados mostram que em geral, os indivíduos desconhecem o caráter permanente do agravo, pois acreditava que o tratamento os levaria à cura do problema renal, além de desconhecerem que o tratamento conservador tem por finalidade diminuir ou manter a lesão renal sem progressão para postergar a necessidade da terapia dialítica substitutiva.

"Por que tem que realizar a HD? (P10); Por que algumas pessoas passam mal durante a hemodiálise? (P06); Durante a hemodiálise vejo sempre algumas pessoas com náuseas, vômitos, umas até sentem frio (P14)"; "Pra mim a hemodiálise é vida, pois estou vivo graças a ela, porque (sic) o medicamento não é só o suficiente para nos ajudar nesse caso. Por isso é importante realizar a hemodiálise (P15)"; "Em caso de transplante, qual a porcentagem de sucesso? Após o transplante necessita continuar o tratamento? (P17); Porque é difícil conseguir o transplante? (P18)".

\section{Discussão/Conclussão}

Os resultados acima apresentados, destacam a necessidade de uma reorganização do processo de trabalho e definição das competências profissionais neste serviço, visto a lacuna emergente apontada para as intervenções educacionais que favoreçam o autoconhecimento, a aceitação, a mudança de comportamento e 0 autocuidado da pessoa com DRC. É sabido que, cabe ao enfermeiro, que trabalha em HD não apenas realizar as funções administrativas e assistenciais, mas também as funções educativas e de pesquisa, principalmente para tentar suprir as falhas provenientes da atenção primária quanto ao diagnóstico e tratamento ${ }^{19}$.

No cotidiano do setor de HD, percebeu-se que diversas pessoas com DRC iniciaram o tratamento em caráter 
emergencial ${ }^{20,21}$. Assim, a experiência do surgimento e a descoberta da DRC se deram de forma desorganizada (no diálogo com P19, 23 e 29), em um processo marcado por experiências caóticas, desconfiança e ambivalência com os serviços de saúde que os assistem (no diálogo com Pll, 12 e 19). 0 estudo de Ribeiro (2013) aponta que a experiência de receber o diagnóstico de DRC, em muitos casos, revela-se como um dos momentos mais difíceis para o doente22-24. Possui experiência entre vários sentimentos, como a angústia diante do desconhecido e o medo frente à possibilidade de sofrimento e morte (no diálogo com P15).

Os participantes revelaram a ausência (20\%) e/ou conhecimento limitado ( $80 \%$ ) sobre as questões que envolvem o que é a DRC e como se desenvolve (23\%), diagnóstico precoce $(8 \%)$, prevenção $(8 \%)$ e tratamento $(33 \%)$, ressaltando constantemente nos discursos a necessidade de identificar as anormalidades o mais cedo possível (no diálogo com P19, 21 e 23). 0 estudo de Oliveira e Soares $(2014)^{25}$ ressalta que, quando há falhas no processo de comunicação para as orientações pode haver comprometimento do entendimento por parte da pessoa com DRC e consequentemente afetar a adesão e o tratamento.

Conseguinte, sem um preparo anterior (informação e educação em saúde), 'são submetidos' aos procedimentos de HD, e isto pode lhe parecer altamente dolorosa e traumática ${ }^{20-22,24}$. E para Faria Rocha e Dos Santos $(2009)^{26}$ e também Carreira e Marcon (2003) ${ }^{27}$, a obrigatoriedade em aceitar a HD como única alternativa para viver gera sentimento de perda da liberdade e da qualidade de vida. Deste modo a aceitação ocorre porque 'o doente' necessita do tratamento, e assim se sente conformado pelo fato de ter uma oportunidade de continuar a viver e vê o tratamento como recomeço, e se adapta aos poucos, à nova vida imposta pela doença ${ }^{28}$. 0 acometimento por uma doença de evolução aguda ou crônica é sempre acompanhado de diversos sentimentos e envolve diversos fatores multicausais, em que frequentemente 0 indivíduo não está preparado para enfrentar num primeiro momento. Pereira e Guedes $(2009)^{29}$ corroboram ao afirmar que essas mudanças geram angústia, sofrimento e que repercutem na relação diária dos doentes. Dificuldades associadas ao processo de comunicação, a falta de informações e 0 desconhecimento de aspectos que envolvem o tratamento, a relação à família e com os demais usuários e membros da equipe de saúde, são pontos importante na adesão, adaptação e até recuperação, em meio às adversidades impostas pela doença e tratamento ${ }^{30}$.
Quando já inseridos no tratamento, as falas denotam dificuldades de aceitação do regime terapêutico, medicamentoso e alimentar, além do distúrbio de imagem corporal, alterações biológicas e psicossociais diversas (no diálogo com P11, 12 E 26), a necessidade de conviver com dores e outras rotinas parecem ser os maiores problemas no dia-a-dia do doente renal em HD (no diálogo com P5, 13 E 24) e também o momento que surge mais dúvidas. A repercussão e a intensidade dos efeitos dessas restrições e mudanças aparecem relacionadas a vários fatores que causam impactos físicos, psicológicos, econômicos e sociais durante a vivência do tratamento ${ }^{24}$. Essa adaptação pode ser facilitada pelo enfermeiro que, por meio do cuidado, planeja intervenções educativas, visando ajudá-los a reaprender a viver com a nova realidade e a sobreviver com a doença renal crônica ${ }^{10,12,16 .}$

A maneira como cada indivíduo vivencia e enfrenta a doença é algo pessoal, influenciado pela estrutura da personalidade, pela capacidade de tolerar frustrações, pelas relações com as pessoas e com seu próprio projeto de vida. Alguns lidam melhor com a enfermidade, procurando informar-se, e são motivados para o tratamento, buscando alternativas para a adaptação. Outros apresentam maiores dificuldades, centrando-se nas emoções e no sofrimento ${ }^{24,32}$.

A proximidade do profissional enfermeiro com as pessoas com DRC, associado à natureza da relação de cuidados, bem com a regularidade e a duração dos tratamentos de HD, proporcionam excelentes oportunidades de monitorizar a adesão, planejar e implementar intervenções que efetivamente ajudem estas pessoas a integrar o regime terapêutico nos seus hábitos diários, dotando-as de conhecimentos, informação e capacitação autônoma, que thes permitam realizar e manter as mudanças necessárias, adaptando-se à sua nova condição de saúde ${ }^{33,34}$. Os relatos dos participantes (40\%) neste estudo apontam o profissional de enfermagem como precursor na prestação de informação relativo à doença, tratamento e autocuidado no serviço de HD.

A função de educador é um dos domínios fundamentais dos cuidados de enfermagem. Tradicionalmente são os enfermeiros que informam as pessoas acerca do que devem esperar da doença, sobre o tratamento ou da intervenção cirúrgica, corrigem as más interpretações e fornecem as informações necessárias para esclarecerem as dúvidas que as pessoas Ihes colocam, tornando um pouco mais familiar, tudo o que a assusta ou the é estranho ${ }^{34}$. É essencial que o profissional de enfer- 
magem conheça tais necessidades e assumam o papel decisivo na educação em saúde. Porém, é sabido das dificuldades que tais profissionais enfrentam para viabilizar tal competência. E apesar dos esforços que os enfermeiros fazem para atender a esta população em "epígrafe", muitas dúvidas ainda fazem parte do cotidiano destas pessoas com DRC ${ }^{25}$.

A intervenção educativa tem que ser oportuna, com metas realistas, e durante longo período de tempo, com reforço frequente ${ }^{35}$. 0 conhecimento deve ser compartilhado, em uma prática dialógica que motivam 0 usuário a ser autônomo. A eficácia das intervenções educacionais só será possível se realizada de forma horizontal, recíproca e em consonância com o novo paradigma da saúde. Nele, o usuário deve ser respeitado como sujeito portador de histórias, crenças e valores ${ }^{36}$.

Os enfermeiros se constituem em um eixo que reúne uma série de ações, como a assistência integral e educação em saúde, envolvendo tanto a equipe de profissionais, quanto aos doentes e os familiares cuidadores $^{37}$. Nessa lógica, o cuidado deve ser precedido de intencionalidade, corresponsabilidade, colaboração e coparticipação de todos envolvidos na assistência ${ }^{38}$.

As narrativas desta pesquisa mostram uma vivência marcada por desafios, revelações, sentimentos, lutas e necessidades de informação, orientação e esclarecimentos. As pessoas com DRC desconhecem o caráter permanente da doença, as complicações, o motivo das restrições e as outras possibilidades quanto ao tratamento. Diante disso, pontua-se que, a abordagem educativa tem suma importância dentro de um serviço de HD, seu objetivo é esclarecer sobre questões da doença, como se desenvolve, as formas de cuidado, métodos terapêuticos, incentivos de práticas de autogestão e possível dificuldade ao longo da jornada de tratamento.

$\mathrm{Na}$ interpretação dos resultados deste estudo, devem-se levar em conta certas limitações, como o tamanho da amostra e a abordagem em um único centro de nefrologia. Sugerimos reaplicação do estudo, com amplitude da amostra e em outras realidades, de modo a verificar compatibilidade dos achados para com outros centros de nefrologia.

É possível afirmar que este estudo é importante para o profissional de enfermagem, uma vez que, identificou lacunas nas suas ações e permitirá uma reorientação da prática com foco em educar, prevenir complicações e promover a saúde destas pessoas com DRC. Enfati- zamos a necessidade de implementação de projetos educacionais neste serviço, com o objetivo ofertar uma melhor evolução da doença, e consequentemente, uma melhor qualidade de vida.

El autor declara que no hay conflicto de interés.

Recibido: 13 marzo 2017

Revisado: 18 mayo 2017

Modificado: 30 noviembre 2017

Aceptado: 5 diciembre 2017

\section{Bibliografía}

1. Salgado Filho N, Brito DJA. Doença renal crônica: a grande epidemia deste milênio. J Bras Nefrol. [Revista na Internet]. 2006 [Acesso 25 janeiro 2015]; 28(3):1-5. Disponível em: http://saudepublica.bvs.br/pesquisa/resource/pt/car-150.

2. ABCDT. Associação Brasileira dos Centros de diálise e transplante. Dia Mundial do Rim agita São Paulo. 2015 [Acesso 23 de junho 2015]; Disponível em: http://www.abcdt.org.br/dia-mundial-dorim-agita-sao-paulo/.

3. KDIGO - Kidney Disease Improving Global Outcomes - work group. Clinical practice guideline for the evaluation and management of chronic kidney disease. Kidney inter. [Revista na Internet]. 2013 [Acesso 25 janeiro 2016]; Suppl(3):1-150. Disponível em: http://www.kdigo.org/clinical_practice_ guidelines/pdf/CKD/KDIG0_2012_CKD_GL.pdf.

4. Dos Santos CA, dos Santos AA, Soares AF. Educação em saúde como instrumento transformador do paciente dialisado: relato de experiência. In Congresso Internacional de Enfermagem. 2017. [Acesso 25 janeiro 2016]; Disponível em: https:// eventos.set.edu.br/index.php/cie/article/viewFile/5970/2017.

5. Sondergaard $H$, Juul S. Self-rated and functioning in patients with chronic renal disease. Dan Med Bul. 2010 [Acesso 30 janeiro 2016]; 57(12):A4220. Disponível em: http://www.dan- 
medj.dk/portal/pls/portal/!PORTAL.wwpob_ page.show?_docname=6316854.PDF.

6. Brasil. Ministério da Saúde. Doença renal crônica atinge $10 \%$ da população mundial. Brasília (DF), 2015 [Acesso 23 junho 2016]; Disponível em: http://www.brasil.gov.br/saude/2015/03/doenca-renal-cronica-atinge-10-da-populacao-mundial.

7. Sesso RCC, Lopes AA, Thomé FS, Lugon JR, Watanabe Y, Santos DR. Diálise Crônica no Brasil Relatório do Censo Brasileiro de Diálise, 2011. J Bras Nefrol [Internet]. 2012 [Acesso 23 junho 2016]; 34(3):272-77. Disponível em: http://www. scielo.br/pdf/jbn/v34n3/v34n3a 09.pdf.

8. Horta HHL, Lopes ML. Complicações decorrentes do tratamento dialítico: contribuição do enfermeiro no cuidado e educação ao paciente. Revista Enfermagem Contemporânea, 2017 [Acesso 23 junho 2016]; 6(2) .Disponível em: https://www5. bahiana.edu.br/index.php/enfermagem/article/ view/1457.

9. Junior MAG, Andreoli MCC, Sardenberg CS, Santos BFC, Neto MC. Diálise no paciente com insuficiência renal crônica: hemodiálise e diálise peritoneal. In: Barros E, Manfro RC, Thomé FS, Gonçalves LFS. Nefrologia: rotinas, diagnóstico e tratamento. Porto Alegre: Artmed, 2006: 424-441.

10. Da Silva Pereira MR. Papéis da enfermagem na hemodiálise. Revista Brasileira de Educação e Saúde, 2013. 3(2):25-36.

11. Burgos E, Meléndez MA, Meza E, Agramon KG, Pereyra MC, Martínez NL. Impacto de uma intervenção destinada a aumentar o conhecimento sobre a doença renal, no início oportuno da terapia de reposição. Rev Soc Esp Enferm Nefrol. 2011; 14 (4): 236-241.

12. Dos Santos I, Rocha RPF, Berardinelli LMM. Necessidades de orientação de enfermagem para o autocuidado de clientes em terapias de hemodiálise. Rev. Bras. Enfermagem. 2011 [Acesso 23 junho 2016]; 64(2):335-42. Disponível em: http:// www.redalyc.org/pdf/2670/267019461018.pdf

13. Stumm EMF, Kirchner RM, Guido LA, Benetti ERR, Belasco AGS, Sesso RCC, et al. Educational nursing intervention to reduce the hyper- phosphatemia in patients on hemodialysis. Rev Bras Enferm. 2017 [Acesso 20 setembro 2017]; 70(1):26-33. Disponível em: http://www.scielo.br/ scielo.php?pid=S0034-71672017000100031\&script=sci_arttext.

14. Stumm EMF, Kirchner RM, Belasco A, Guido LA, Barbosa DA. Validation of a manual for patients with hyperphosphatemia. J Nurs UFPE. 2013;7(9):5485-9.

15. Queiroz MV0, Dantas MCQ, Ramos IC, Jorge MSB. Tecnologia do cuidado ao paciente renal crônico: enfoque educativo-terapêutico a partir das necessidades dos sujeitos. Texto \& Contexto Enferm. 2008;17(1):55-63.

16. De Lima MA, Sousa GR, De Sousa AM, Felipe GF, De Oliveira ASS, Formiga LMF. Educação em saúde para pacientes em hemodiálise. Revista de enfermagem UFPE on line-ISSN: 1981-8963, 2014 [Acesso 20 setembro 2017]; 8(6): 15101515. Disponível em: https://periodicos.ufpe.br/ revistas/revistaenfermagem/article/view/9839.

17. Minayo MCS. 0 desafio do conhecimento: pesquisa qualitativa em saúde. 14.ed. São Paulo: Hucitec, 2014.

18. BRASIL. Conselho Nacional de Saúde. Resolução 466, de 12 de dezembro de 2012. Aprova as diretrizes e normas regulamentadoras de pesquisa envolvendo seres humanos. Diário Oficial da União, Brasília, n. 12, 13 de junho de 2013 - seção 1, página 59.

19. Fava $S M C L$, Oliveira AAD, Vitor EM, Damasceno DD, Libânio SIC. Complicações mais frequentes relacionadas aos pacientes em tratamento dialítico. Reme: Rev. Min. Enf.; 2010 [Acesso 20 setembro 2017]; abr-jun; 10(2): 145-150. Disponível em: http://www.reme.org.br/artigo/detalhes/399.

20. Godinho TM, Lyra TG, Braga PS, Queiroz RAD, Alves JA, Kraychete AC et al. Perfil do paciente que inicia hemodiálise de manutenção em hospital público em Salvador, Bahia. J Bras Nefrol, 2006. 28(2): 96-103.

21. Sampaio RMM, Coelho M0, Pinto FJM, Ostema EPR. Perfil epidemiológico de pacientes nefropatas e as dificuldades no acesso ao tratamento. Revista Brasileira em Promoção da Saúde, 
2013 [Acesso 20 setembro 2017]; 26(1):95101. Disponível em: http://www.redalyc.org/ html/408/40827988014/.

22. Ribeiro CDS, Alencar CSM, Feitosa MCD, Mesquita MADSB. Percepção do portador de doença renal crônica sobre o tratamento hemodialítico. Revista Interdisciplinar, 2013 [Acesso 20 setembro 2017]; 6(3):36-44. Disponível em: https:// revistainterdisciplinar.uninovafapi.edu.br/index. php/revinter/article/view/91.

23. Rudnicki T. Preditores de qualidade de vida em pacientes renais crônicos. Estud psicol. (Campinas). $2007 ; 24(3)$.

24. Rocha MLD, Vieira SDS, Braga SD0, Poveda VDB, Sanchez EH. Início do Tratamento hemodialítico: qualidade de vida, sentimentos e dificuldades. Journal of Nursing UFPE on line, 2009. $3(2): 223-229$.

25. Oliveira AM; Soares E. Uma Comunicação como Importante Ferramenta nas Orientações em uma Unidade de Hemodiálise: um estudo reflexivo. Saúde \& Social Transformação / Saúde e Mudança Social, 2014. [Acesso 20 setembro 2017]; 5 (3): 118-123. Disponível em: http://www.redalyc.org/ articulo.oa? id=265335335017.

26. Faria Rocha RP; Dos Santos I. Necessidades de autocuidado entre clientes com doença renal crônica: revisão integrativa de literatura. Revista de Pesquisa Cuidado é Fundamental Online, 2009 [Acesso 20 setembro 2017]; 1(2). Disponível em: http:// www.redalyc.org/html/5057/505750816030/.

27. Carreira L; Marcon SS. Cotidiano e trabalho: concepções de indivíduos portadores de insuficiência renal crônica e seus familiares. Revista Latino-Americana de Enfermagem, 2003 [Acesso 20 setembro 2017]; 11(6):823-831. Disponível em: http://www.revistas.usp.br/rlae/article/view/1837.

28. Souza Terra F, Costa AMDD, Ribeiro CCS, Nogueira CS, Prado JP, Costa MD. 0 portador de insuficiência renal crônica e suas dependências ao tratamento hemodialítico: compreensão fenomenológica. Rev Bras Clin Med. 2010 8. [Acesso 2 junho 2017]; (4):306-10. Disponível em: http:// www.sbcm.org.br/revistas/RBCM/RBCM-201004.pdf\#page $=17$.
29. Pereira LD, Guedes MVC. Hemodiálise: a percepção do portador renal crônico. Cogitare Enfermagem. 2009 [Acesso 02 junho 2017]; 14(4):689-95. Disponível em: http://www.redalyc.org/html/4836/483648977015/.

30. Pietrovsk V, Dall'agnol CM. Situações significativas no espaço-contexto da hemodiálise: o que dizem os usuários de um serviço?. Revista Brasileira de Enfermagem, 2006 [Acesso 02 junho 2017]; 59(5). Disponível em: http://www.redalyc. org/html/2670/267019619007/.

31. Matarán Robles EM, Aguilar García R, Muñoz Becerra M. Incidencia y tipo de efectos adversos durante el procedimiento de hemodiálisis. Enfermería Nefrológica [revista na Internet]. 2013. [Acesso 17 setembro 2016]; 16(1): 36-40. Disponível em: http://www.revistaseden.org/imprimir.aspx?idArticulo $=4424170094091095096424170$.

32. Madalosso FD, Mariotti MC. Terapia ocupacional e qualidade de vida de pessoas com insuficiência renal crônica em hemodiálise. Cad Bras Ter Ocup. 2013 [Acesso 20 agosto 2016]; 21(3):51120. Disponível em: http://dx.doi.org/10.4322/ cto.2013.053.

33. Fernandes M; Cruz L. Adesão ao regime terapêutico da pessoa em início de hemodiálise-intervenção do enfermeiro. CIAIQ, 2017. [Acesso 20 agosto 2016]; Disponível em: http://proceedings. ciaiq.org/index.php/ciaiq2017/article/view/1251.

34. Machado, MMP. Adesão ao Regime Terapêutico: Representações das pessoas com IRC sobre o contributo dos enfermeiros. Dissertação de mestrado em Educação (especialização em Educação para a Saúde). 2009.

35. Edwards, M. Supporting patients after a diagnosis with diabetes. Practice Nursing, 2009 [Acesso 20 agosto 2016]; 20(4):185-187. Disponível em: https://www.magonlinelibrary.com/doi/ abs/10.12968/pnur.2009.20.4.41206?journalCode=pnur.

36. Gonzalez CM, Teixeira MLO, Castelo Branco SEM. Cuidado educativo compartilhado: estratégia de ação da enfermagem junto a usuários com insuficiência renal crônica. Rev baiana de enfermagem. 2017;31(3):e17536. 
37. Tejada-Tayabas LM, Partida-Ponce KL, Hernández-Ibarra LE. Atendimento hospitalar-domiciliar coordenado para pacientes renais em hemodiálise sob a perspectiva do pessoal de enfermagem. Revista latino-americana de enfermagem, 2015. 23(2): 225-233.

38. Vanderboom CE, Thackeray NL, Rhudy LM. Key factors in patient-centered care coordination in ambulatory care: Nurse care coordinators' perspectives. Appl Nurs Res [Revista da Internet]. 2015 [Acesso 17 setembro 2016]; 28(1):18-24. Disponível em: https://www.ncbi.nlm.nih.gov/pub$\mathrm{med} / 24746283$.

Este artículo se distribuye bajo una Licencia Creative Commons Atribución-NoComercial 4.0 Internacional. https://creativecommons.org/licenses/by-nc/4.0/

Open Access (c) (7) (8) 